\title{
Alternative Method of Spectroscopy of Alkali Metal RGB
}

\author{
Ricardo Gobato $^{1}$ *, Manuel Simões Filho ${ }^{2}$ \\ ${ }^{1}$ State Secretary of Education of Paraná, Laboratory of Biophysics and Molecular Modeling, J. C. State College, High School and Integral, Bela \\ Vista do Paraíso, Paraná, Brazil \\ ${ }^{2}$ State University of Londrina, Department of Physics, Londrina, Paraná, Brazil
}

Email address:

ricardogobato@seed.pr.gov.br (Ricardo G.), simoes@uel.br (Manuel S. F.)

*Corresponding author

\section{To cite this article:}

Ricardo Gobato, Manuel Simões Filho. Alternative Method of Spectroscopy of Alkali Metal RGB. Modern Chemistry. Vol. 5, No. 4, 2017, pp. 70-74. doi: 10.11648/j.mc.20170504.13

Received: June 11, 2017; Accepted: June 23, 2017; Published: October 18, 2017

\begin{abstract}
Spectroscopy is a technique for collecting physicochemical data through the transmission, absorption or reflection of incident radiant energy in a sample. In this work we used a didactic and alternative method for spectroscopy. Our work is used in common low cost and easy access devices that have a CCD reader. The idea is a set of devices, such as a cell phone, which contains an optical CCD reader, where these equipment materials, materials, compounds, simplifying the image obtained by these optical devices, analyzed and characterized. Our technique consists of decoding of the pixels of the .bmp images, decoding and quantifying their RGB color channels. These images were obtained from shooting flames produced by alkali metals, lithium, sodium, potassium, rubidium and cesium. We conclude that it is possible to do a spectroscopic analysis using our technique.
\end{abstract}

Keywords: Alkali Metal, Caesium, Lithium, RGB, Rubidium

\section{Introduction}

Spectroscopy is a technique for collecting physicochemical data through the transmission, absorption or reflection of incident radiant energy in a sample. It is much employed to be used in their spectra, which is difficult to access equipment because of its high cost, found in research surveys. [1, 2, 3]

Spectroscopic studies were central to the development of quantum mechanics and included Max Planck's explanation of blackbody radiation, Albert Einstein's explanation of the photoelectric effect and Niels Bohr's explanation of atomic structure and spectra. Spectroscopy is used in physical and analytical chemistry because atoms and molecules have unique spectra. As a result, these spectra can be used to detect, identify and quantify information about the atoms and molecules. Spectroscopy is also used in astronomy and remote sensing on earth. Most research telescopes have spectrographs. The measured spectra are used to determine the chemical composition and physical properties of astronomical objects (such as their temperature and velocity). [4, 5]

Modern implementations of atomic spectroscopy for studying visible and ultraviolet transitions include flame emission spectroscopy, inductively coupled plasma atomic emission spectroscopy, glow discharge spectroscopy, microwave induced plasma spectroscopy, and spark or arc emission spectroscopy. Techniques for studying x-ray spectra include X-ray spectroscopy and X-ray fluorescence. [4, 5]

Our idea a set of devices, such as a cell phone, which contains an optical $\mathrm{CCD}^{1}$ reader, where these equipment materials, materials, compounds, simplifying the image obtained by these optical devices. As filming obtained by optical CCD reader theses hardware, form decoded and separated into their quantified RGB color channels.

Our technique consists of the analysis of the pixels of the images of the .bmp images, decoding and quantifying their RGB color channels. [6] These images were obtained from shooting flames produced by alkali metals, lithium, sodium, potassium, rubidium and caesium.

The alkali metals are a group (column) in the periodic table consisting of the chemical elements lithium (Li), Sodium $(\mathrm{Na})$, Potassium (K), Rubidium (Rb), Caesium (Cs) and Francium (Fr). This group lies in the $s$ block of the periodic table of elements as all alkali metals have their outermost electron in

1 A Charge-Coupled Device (CCD) is a device for the movement of electrical charge, usually from within the device to an area where the charge can be manipulated. 
an $s$ orbital: this shared electron configuration results in them having very similar characteristic properties. Indeed, the alkali metals provide the best example of group trends in properties in the periodic table, with elements exhibiting well characterized homologous behavior. [2, 7]

The Figure (1) represents of a black image of $100 \times 100$ pixels, that is, 10,000 pixels. As an example a file that has 30,054 bytes of size, of which 54 bytes are of file identification as the "family" bmp, corresponds to 30,000 bytes, dividing by 3 , we have 10,000 pixels, if it is of quadrangular dimensions.

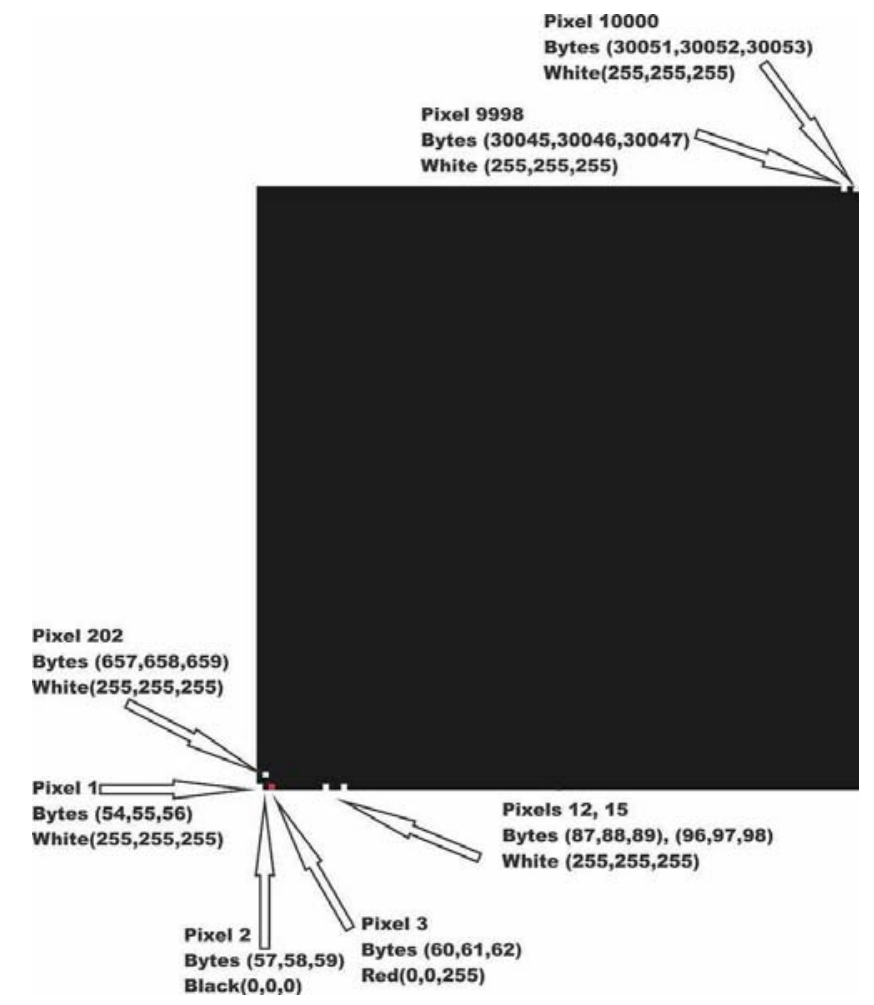

Figure 1. Representation of a black image of $100 \times 100$ pixels, i.e., 10,000 pixels. We will have $100 \times 100$ pixels, which correspond to $300 \times 100$, Table (2.1). Decimal and Hexadecimal representation of some bytes. Colors in combination of three pixels. [8,9].

\section{History}

The RGB color model is based on the Young-Helmholtz theory of trichromatic color vision, developed by Thomas Young and Hermann Helmholtz in the early to mid-nineteenth century, and on James Clerk Maxwell's color triangle that elaborated that theory 1,860 . $[6,7]$

One common application of the RGB color model is the display of colors on a cathode ray tube (CRT), liquid crystal display (LCD), plasma display, or organic light emitting diode (OLED) display such as a television, a computer's monitor, or a large scale screen. Each pixel on the screen is built by driving three small and very close but still separated RGB light sources. At common viewing distance, the separate sources are indistinguishable, which tricks the eye to see a given solid color. All the pixels together arranged in the rectangular screen surface conforms the color image. [6, 7]

Photographic digital cameras that use a CMOS or CCD image sensor often operate with some variation of the RGB model. In a Bayer filter arrangement, green is given twice as many detectors as red and blue (ratio 1:2:1) in order to achieve higher luminance resolution than chrominance resolution. The sensor has a grid of red, green and blue detectors arranged so that the first row is RGRGRGRG, the next is GBGBGBGB, and that sequence is repeated in subsequent rows. For every channel, missing pixels are obtained by interpolation in the demos aicing process to build up the complete image. Also, other processes used to be applied in order to map the camera RGB measurements into a standard RGB color space as sRGB. $[6,7]$

\section{Pixels and the Bmp Image}

Pixel (the agglutination of Picture and Element). [5] Every three bytes equals one pixel in a bmp file. Therefore each color is defined by a set of three bytes, one pixel. On a color monitor each pixel is composed of a set of 3 bytes ( 3 colors): red (R), green $(\mathrm{G})$, and blue (B), the RGB standard used here. RGB is the abbreviation for the additive color system consisting of Red (Red), Green (Green) and Blue (Blue). In the best monitors each of these points is capable of displaying 256 different shades (equivalent to 8 bits) and combining shades of three dots $(256 \mathrm{x}$ $256 \times 256)$ it is then possible to display just over 16.7 million different colors (exactly 16,777,216). At 640 x 480 resolution we have 307,200 pixels, approximately 0.31 megapixel, at 800 x 600 we have 480,000 pixels, at 1024 x 768 we have 786,432 pixels and so on. In a bmp file the image is determined from byte number 54 . From byte 0 to 53 is machine identification of the bmp file, such as size, image quality, etc. The image in Figure (1), the sequence of bytes 54,55 , and 56 , matches on your screen as the lower left corner. The location of each pixel is from left to right to the end of the screen, sequencing on a line just above, from left to right, and so on, from left to right and from bottom to top respectively. $[8,9,10,11]$

Table 1. The table above shows a sample of the three RGB channels, at their intensities for some colors in decimal e hexadecimal. One can notice an $R G B$ matrix, $A_{i j}=A_{3 \times 3}=255 . I_{3 \times 3}$.

\begin{tabular}{lllll}
\hline Color & & Decimal & Hexadecimal \\
\hline & Red & Green & Blue & \\
White & 255 & 255 & 255 & FFFFFF \\
Black & 0 & 0 & 0 & 000000 \\
Red (R) & 255 & 0 & 0 & FF0000 \\
Green (G) & 0 & 255 & 0 & 00FF00 \\
Blue (B) & 0 & 0 & 255 & 0000FF \\
Yellow & 255 & 255 & 0 & FFFF00 \\
Magenta & 255 & 0 & 255 & FF00FF \\
Violet & 238 & 130 & 238 & EE82EE \\
Plum & 221 & 160 & 221 & DDA0DD \\
Purple & 128 & 0 & 128 & 800080 \\
Teal & 0 & 128 & 128 & 008080 \\
Silver & 192 & 192 & 192 & C0C0C0 \\
Gold & 255 & 215 & 0 & FFD700 \\
Khaki & 240 & 230 & 140 & F0E68C \\
\hline
\end{tabular}

\section{Materials and Methods}

We present the data of this spectroscopy didactic technique 
for decoding of each image was obtained through the program ImageJ, [12] a software available for free access on the internet. In our experiment several events were filmed, and so we developed a software for the decoding of each image, as well as the end result of the quantity of each color of the RGB channels, from 0 to 255 .

Only one single image of each event of all images is shown. The best images were separated, which presented minor imperfections of manipulation of a common user. A fact that is the goal of the technique, that an ordinary user takes a single photograph, or make the filming and this device can determine what this image is about.

The images were obtained through the Learn Chemistry, of Royal Society of Chemistry [13], of these films were separated their due frames with output of image of .jpg and converted to the format of work .bmp, Standard RGB.

The software was developed in Delphi language, [14] where source algorithms for converting .jpg images to .bmp and separation of RGB color channels were also obtained from free access on the internet.

The films of each of the studied chemical elements were downloaded from Learn Chemistry, Royal Society of Chemistry. $[13,15]$ These generated a significant amount of images of dimensions 1920 x 1080, 2,073,600 pixels, in .jpg format, being corverted to the .bmp format [14], from which the number of 456, 224, 273, 105 and 18 numbers were selected for the elements Lithium, Sodium, Potassium, Cesium and Rubidium, respectively. The graphic was built for each RGB color channel separately, through the normalized average of numbers selected of frames of $1920 \times 1080$ image, files in .bmp format, after the previous image conversion.

\section{Results}

The Figures (1) (2) and (3) represent a ternary graph in percent for the intensity of each RGB color (Red, Green and Blue) and each has its own characteristic.

Each graph is normalized to the intensity of each RGB color channel from 0 to 255 .

The graphs of Figures (1) (2) and (3) show the average normalized frequency of the intensities of each RGB (Red, Green and Blue) color ranging from 0 to 255 , of the studied alkali metals: lithium, sodium, potassium, rubidium and cesium.

The graph of Figure (1) shows the normalized average frequency of red color intensity varying from 0 to 255 , of the alkali metals studied: Lithium (Li), Sodium (Na), Potassium (K), Rubidium ( $\mathrm{Rb}$ ), Caesium (Cs). Likewise Figure (2) for the color Green and Figure (3) for the color Blue.

We can verify the intrinsic characteristic of each chemical element, presented by its characteristic curve.

We can identify the ternary graph, the alkali metals studied: lithium, sodium, potassium, rubidium and cesium, using this method separation of the RGB color channels, in a quantitative analysis of the pixels of the .bmp images.

In Figure (4) a selected image of each chemical element studied is visualized.

The Figure (5) show the ternary graphs, $(\% \mathrm{R}+\% \mathrm{G}+\% \mathrm{~B})$
$=1$, for each chemical element studied: lithium, sodium, potassium, rubidium and cesium, as well as its characteristic curve for each RGB color channel. In the ternary graph the characteristic of each RGB spectrum of each one of the elements studied in this work is well elucidated.

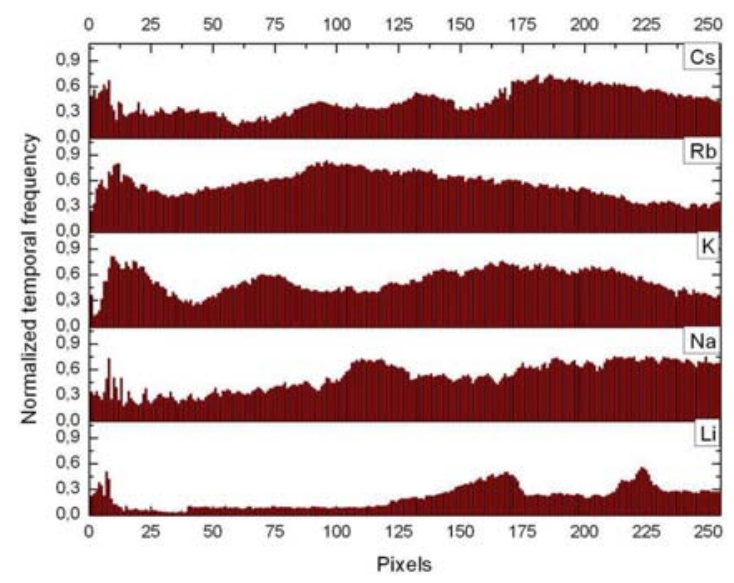

Figure 1. The graphs show the average normalized frequency of the intensities of Red color ranging from 0 to 255, of the studied alkali metals: Lithium (Li), Sodium (Na), Potassium (K), Rubidium (Rb) and Caesium (Cs).

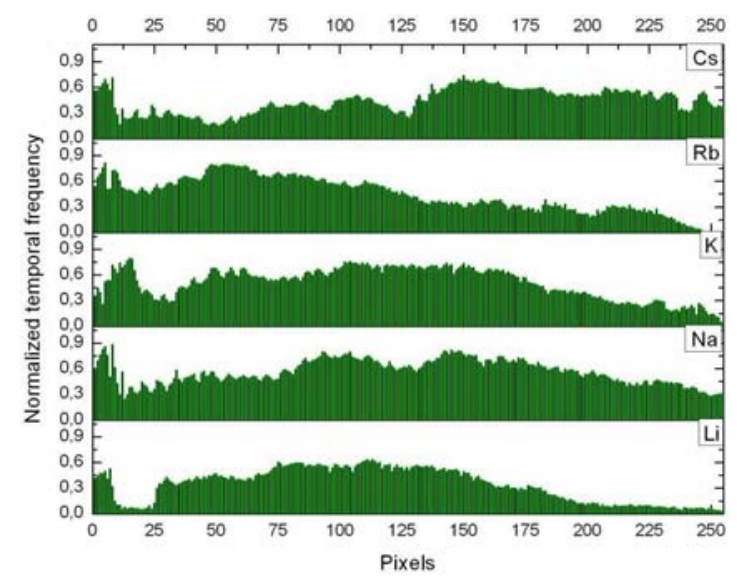

Figure 2. The graphs show the average normalized frequency of the intensities of Green color ranging from 0 to 255, of the studied alkali metals: Lithium (Li), Sodium (Na), Potassium (K), Rubidium (Rb) and Caesium (Cs).

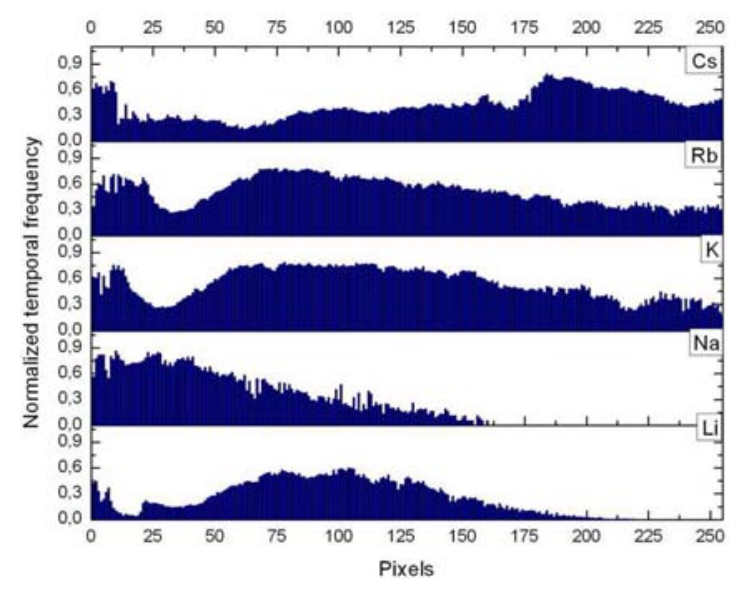

Figure 3. The graphs show the average normalized frequency of the intensities of Blue color ranging from 0 to 255, of the studied alkali metals: Lithium (Li), Sodium (Na), Potassium (K), Rubidium (Rb) and Caesium (Cs). 

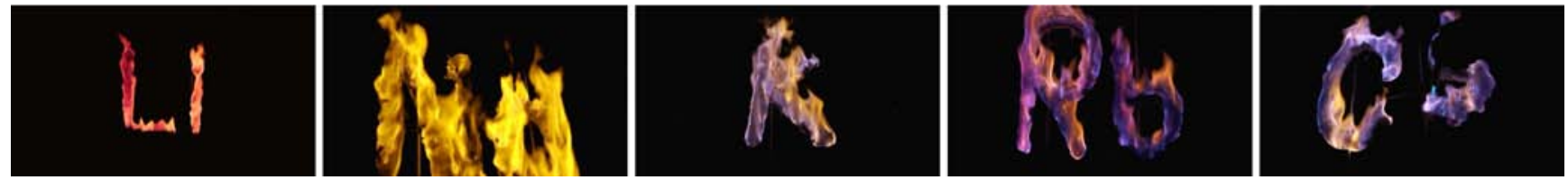

Figure 4. Selected images of the flame of the five alkali metals: Lithium Flame, Sodium Flame, Potassium Flame, Rubidium Flame and Caesium Flame. $[13,15]$
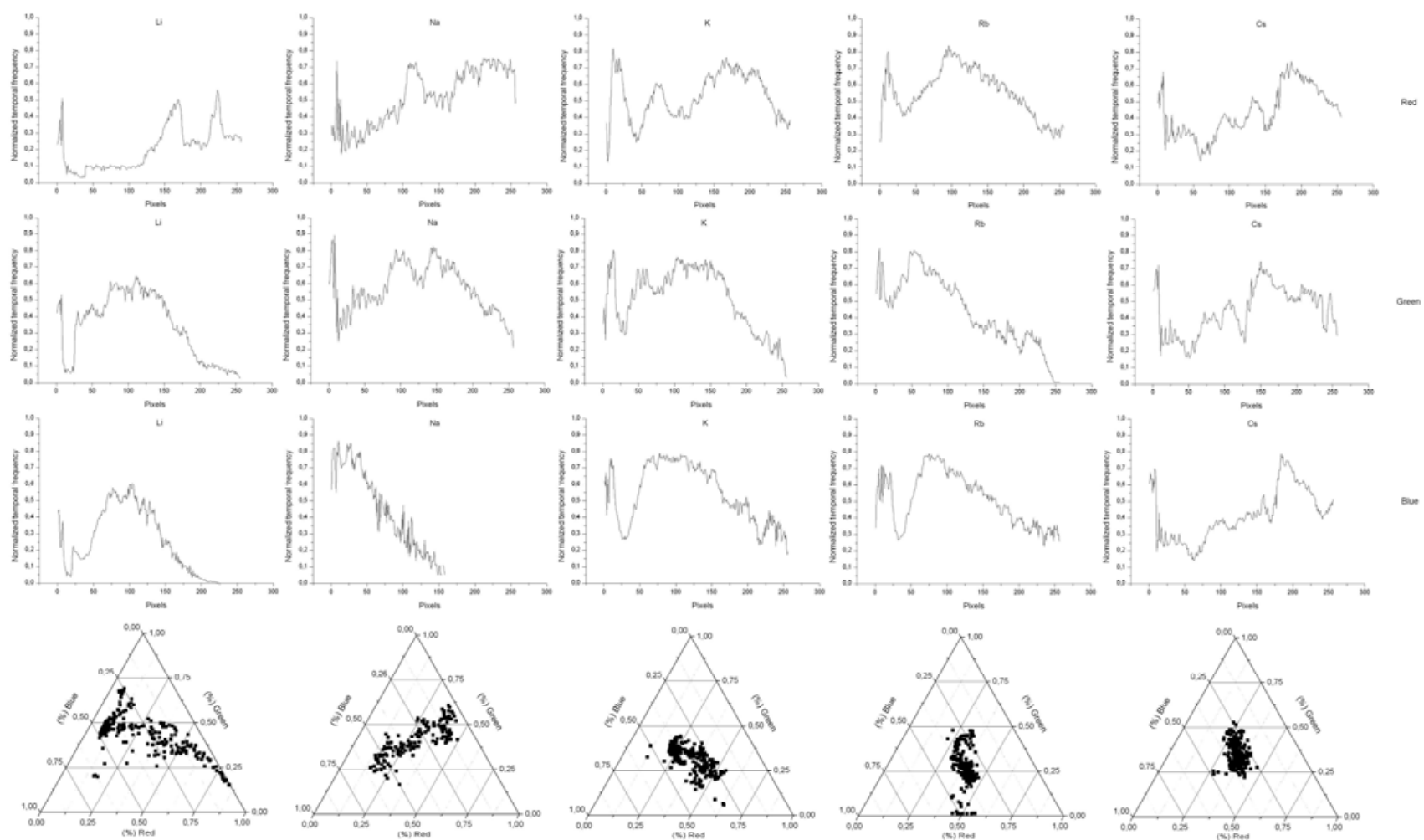

Figure 5. Ternary graphs for each chemical element studied: lithium, sodium, potassium, rubidium and cesium, as well as its characteristic curve for each RGB color channel. In the ternary graph the characteristic of each RGB spectrum of each one of the elements Lithium (Li), Sodium (Na), Potassium (K), Rubidium (Rb) and Caesium (Cs).

\section{Conclusions}

We can verify the intrinsic characteristic of each chemical element studied: Lithium (Li), Sodium (Na), Potassium (K), Rubidium $(\mathrm{Rb})$ and Caesium $(\mathrm{Cs})$, using this method separation of the RGB color channels.

By doing a more detailed study one can obtain characteristic mathematical parameters and graphs that can identify things, materials, compounds, in others, using this technique of spectroscopic analysis by separating RGB color channels from .bmp images.

The obtained results could be compared with spectroscopic techniques. They results be clear, but need to new studies, as a comparison with spectroscopic techniques and an amplification of the range of compounds analyzed. Our work has shown that any equipment containing a CCD can, with appropriate software, analyze and identify chemical particles by analyzing the obtained images.

\section{References}

[1] R. E. Newnham. Properties of materials. Anisotropy, Simmetry, Structure. Oxford University press, New York, 2005.

[2] General Chemistry Principles, Patterns, and Applications. Saylor.org.

[3] B. D. Mistry. A Handbook of Spectroscopic Data CHEM ISTRY. Oxford Book Company. Jaipur, India, Oxford Book Company. Jaipur, India., 2009.

[4] W. S. Struve. Fundamentals of Molecular Spectroscopy. Department of Chemistry. Iowa State University, Ames, Iowa. John Wiley and Sons, 1989.

[5] G. Gauglitz and T. Vo-Dinh. Handbook of Spectroscopy. KGaA, Weinheim, Wiley-VCH Verlag GmbH \& Co. 2003. ISBN 3-527-29782-0.

[6] Hsien Che Lee. Introduction to Color Imaging Sience. United States of America by Cambridge University Press, New York, Cambridge University Press. The Edinburgh Building, Cambridge, UK, 2005. 
[7] CC BY NC SA 3.0. Creative Commons. Wikipedia, The Free Encyclopedia, May 2016. CC BY NC SA 3.0.

[8] M. Simões F. and R. Gobato. Espectroscopia por Mapeamento RGB de Fontes Primárias de Luz. In XIX Semana da Física. Simpósio Comemorativo dos 40 Anos do Curso de Física da Universidade Estadual de Londrina. Univer sidade Estadual de Londrina, Sep. 15- 19, 2014.

[9] M. Simões F. and R. Gobato. Espectroscopia por Mapeamento RGB de Fontes Primárias de Luz. viXra.org. Condensed Matter, p. 366, Jan. 06, 2017. viXra:1701.0306.

[10] K. Roth. Chem. Unserer Zeit., 37:424-429, 2003.
[11] M. Faraday. The Chemical History of a Candle. Dover, Mineola, USA, 2002.

[12] W. Rasband. ImageJ 1.51 i Software.

[13] Royal Society of Chemistry. Learn chemistry. Enhancing learning and teaching. Royal Society of Chemistry 2017, Nov. 2014.

[14] Planeta Delphi. Tudo sobre programação Delphi, Jan. 12, 2017.

[15] CC BY NC SA 2.0. Creative Commons, Jan. 2017. CC BY NC SA 3.0. 